\title{
Comparison of SCAphoid fracture osteosynthesis by MAGnesium-based headless Herbert screws with titanium Herbert screws: protocol for the randomized controlled SCAMAG clinical trial
}

Sören Könneker ${ }^{1 * \dagger}$, Katja Krockenberger ${ }^{2 \dagger}$, Claudia Pieh ${ }^{3}$, Christian von Falck ${ }^{4}$, Bernard Brandewiede ${ }^{2}$, Peter M. Vogt ${ }^{1+}$, Martin H. Kirschner ${ }^{3,5+}$ and Andreas Ziegler ${ }^{6,7^{* \dagger}}$ (D)

\begin{abstract}
Background: Scaphoid fractures are the most common carpal fractures. They often need to be treated by surgery, where the use of a compression screw is the globally accepted gold standard. Surgeons may choose between different implant materials including titanium alloys, which remain in the body or are removed after healing. An alternative are biodegradable magnesium-based implants. Properties of magnesium alloys include high stability, osteoconductivity, potential reduction of infections and few artifacts in magnetic resonance imaging (MRI). The aim of this trial is to demonstrate non-inferiority of magnesium-based compression screws compared with titanium Herbert screws for scaphoid fractures.

Methods: The trial is designed as a multicenter, blinded observer, randomized controlled parallel two-group post market trial. Approximately 190 patients will be randomized (1:1) with stratification by center either to titanium or magnesium-based compression screws. Follow-up is 1 year per patient. Surgical procedures and aftercare will be performed according to the German treatment guideline for scaphoid fractures. The first primary endpoint is the patient-rated wrist evaluation (PRWE) score after 6 months. The second primary endpoint is a composite safety endpoint including bone union until 6 months, no adverse device effect (ADE) during surgery or wound healing and no serious ADE or reoperation within 1 year. The third primary endpoint is the difference in change MRI artifacts over time. Non-inferiority will be investigated for primary endpoints 1 (t-test confidence interval) and 2 (Wilson's score interval) using both the full analysis set (FAS) and the per protocol population at the one-sided 2.5\% test-level. Superiority of magnesium over titanium screws will be established using the FAS at the two-sided 5\% test-level (Welch test) only if non-inferiority has been established for both primary endpoints. Secondary endpoints include quality of life.

(Continued on next page)
\end{abstract}

\footnotetext{
*Correspondence: koenneker.soeren@mh-hannover.de; ziegler@statsol.de

†'Sören Könneker and Katja Krockenberger are shared first authorship.

${ }^{\dagger}$ Peter M. Vogt, Martin H. Kirschner and Andreas Ziegler are shared last authorship.

${ }^{1}$ Department of Plastic, Aesthetic, Hand and Reconstructive Surgery, Hanover Medical School (MHH), Carl-Neuberg-Straße 1, 30625 Hannover, Germany

${ }^{6}$ StatSol, Moenring 2, 23560 Lübeck, Germany

Full list of author information is available at the end of the article
}

(c) The Author(s). 2019 Open Access This article is distributed under the terms of the Creative Commons Attribution 4.0 International License (http://creativecommons.org/licenses/by/4.0/), which permits unrestricted use, distribution, and reproduction in any medium, provided you give appropriate credit to the original author(s) and the source, provide a link to the Creative Commons license, and indicate if changes were made. The Creative Commons Public Domain Dedication waiver (http://creativecommons.org/publicdomain/zero/1.0/) applies to the data made available in this article, unless otherwise stated. 
(Continued from previous page)

Discussion: This study will inform care providers whether biodegradable magnesium-based implants are non-inferior to standard titanium Herbert screws for the treatment of scaphoid fractures in terms of wrist function and safety. Furthermore, superiority of magnesium-based implants may be demonstrated using MRI, which is used as surrogate endpoint for screw degradation.

Trial registration: DRKS, DRKS00013368. Registered Dec 04, 2017.

Keywords: Herbert screw, Magnesium alloy, Magnetic resonance imaging, Non-inferiority, Patient-rated wrist evaluation, Quality of life, Scaphoid fracture, Titanium alloy

\section{Background}

The fracture of the scaphoid is the most common carpal fracture accounting for 2 to $7 \%$ of all fractures [1], and young men between 20 and 29 years are predominantly affected [2]. This has an economic impact on the society caused by the absence from work. Therefore, the goals of treatment are to avoid long immobilization, bone healing disorders i.e., pseudarthrosis of the scaphoid and to gain normal wrist function.

The probability for bone healing mainly depends on displacement and location of the fracture and decreases substantially if the fracture gap width exceeds $2 \mathrm{~mm}$ [3]. Surgical treatment is generally recommended for unstable fractures, which include fractures with displacement $\geq 1 \mathrm{~mm}$ or $\geq 2 \mathrm{~mm}$, comminuted fractures, perilunate fracture-dislocation and all fractures in the proximal third because of a long immobilization time and a high risk for pseudarthrosis [4]. Conservative treatment is often recommended for stable fractures [5]. Specifically, the German treatment guideline for scaphoid fractures [6] recommends conservative treatment of tubercle fractures (type A1) and non-dislocated transverse fractures in the middle or distal third (type A2) for the classification scheme by Herbert and modified by Krimmer et al. [4]. Recommendations for operative treatment have, however, been expanded in recent years to include non-displaced waist fractures to avoid long periods of immobilization and by that resulting joint stiffness [5], and the German treatment guideline recommends minimal invasive surgery for A2 fractures.

Headless compression screws are considered to be particularly well suited for surgical therapy of recent fractures [6], which can be inserted through both palmar and dorsal approaches. Most frequently, non-biodegradable metallic implants are used, specifically titanium or steel alloys. However, surgeons may also choose absorbable implants, e.g., polymer-based implants [7] and magnesiumbased implants $[8,9]$, which have the clear advantage that they degrade over time so that no second surgery is required for implant removal $[10,11]$. Screw removal rates between 8 and 14\% have been reported for various indications in the literature [12-14]. A major advantage of magnesium-based implants over polymer implants is their higher stability $[8,9]$. They also have osteoconductive properties [9] and are supposed to inhibit infections [9].

Few artifacts in magnetic resonance imaging and computed tomography scans

Furthermore, magnesium-based implants produce almost no artifacts in MRI or CT even when imaging was done immediately after implantation $[9,15]$. In contrast, titanium screws generally create interference. On the $3 \mathrm{~T}$ scanner employed by Sonnow et al. [15], the largest difference in artifacts between magnesium-based and titanium implants was observed when imaging was done with the T1w turbo spin echo (TSE) sequences. Fewer artifacts were also observed for the proton density weighted (PDw) TSE and PDw TSE metal-artifact reduction (WARP) sequences. However, differences were less pronounced.

Magnesium-based implants thus better facilitate postoperative follow-up. In addition, since magnesium-based implants are biodegradable, a further reduction of artifacts over time is expected. Change of artifact size over time may thus serve as surrogate marker for screw degradation.

\section{Magnesium-based compression screw}

MAGNEZIX $^{\bullet}$ CS of Syntellix AG are magnesium-based Herbert screws. The alloy is based on the MgYREZr/ WE43 system and contains more than $90 \%$ magnesium and, in addition, yttrium, rare earth metal and zirconium. It is free of aluminium, a well-known neurotoxin [16]. MAGNEZIX ${ }^{\circ}$ CS has market approval for the European Union, countries belonging to the European Free Trade Association and 21 additional countries worldwide (as of July 31, 2018). The small CS 2.0 is not cannulated, while CS 2.7 and CS 3.2 are cannulated to allow insertion of a guide wire (Fig. 1). All screw models have a self-cutting tip to ease insertion. During screw implantation, specially modified instruments are used.

\section{Radiolucent zone around any part of the implant}

Radiolucent zones around magnesium-based implants have been described in the literature $[17,18]$. Although 


\section{$=$
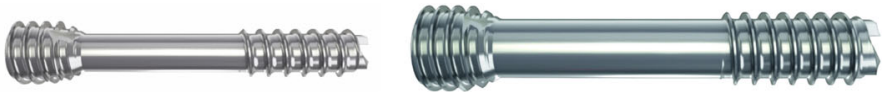

Fig. 1 Magnesium-based compression screws to be used in this trial. From left to right: MAGNEZIX® ${ }^{\circledR}$ 2.0, MAGNEZIX ${ }^{\circledR}$ CS 2.7, MAGNEZIX® CS 3.2

this may be visually inconvenient, this effect is only short-term, it does not affect bone healing and disappears automatically over time. Experiences from laboratory testing animal studies have shown the disappearance of the screw within 1 year $[17,18]$, and in a clinical trial it was degraded after 3 years [19].

In summary, radiolucent zones are expected during the degradation of the screw because of the material properties of magnesium and its degradation via corrosion. Consequently, this effect is not taken account as a screw-related complication.

\section{Breakage of magnesium-based screws in the healing process}

When the healing process is well advanced, MAGNE$\mathrm{ZIX}^{\circ}$ implants will be recognized as being deformed in diagnostic imaging caused by the degeneration process of the implant. In this process, these implants did not break because of a lack of stability, but they were degrading as intended, while the bone continued to heal and gradually bore higher load capacity.

In summary, the breakage of MAGNEZIX ${ }^{\circ}$ CS during the healing process may occur due to the degradation process. Consequently, this effect is not considered a screw-related complication.

\section{Clinical evidence of magnesium-based screws}

In an RCT, biodegradable magnesium-based screws were not inferior to titanium screws for the treatment of mild hallux valgus deformities [20]. Primary endpoint of this trial was the absolute difference between the distal metatarsal articular angle (DMAA) measured 6 months postsurgery and immediately post-surgery. The authors also measured the American Orthopedic Foot and Ankle Society (AOFAS) score for hallux, visual analog scale (VAS) for pain assessment and range of motion (ROM) of the first metatarsophalangeal (MTP-IP) joint. The authors observed comparable values between titanium and magnesium-based screws for these outcome variables. In addition, no foreign body reactions, osteolysis and systemic inflammatory reactions were detected. During the implants' absorption process, no increase of the laboratory values of electrolytes including magnesium or other components or degradation products of the magnesiumbased screw were found [20]. Three-year follow-up data to this trial have been reported [19].

Magnesium-based compression screws are meanwhile used in several indications, e.g., for the treatment of hallux valgus [19-22], malleolar fractures [23, 24], ankle joint fractures [25], in the treatment of fibula fractures [25], scaphoid fractures [26, 27] or radial head fractures [28].

\section{The need for a trial}

Windhagen et al. [20] focused on elective foot surgery. However, an RCT for the use of magnesium-based screws is lacking in fractures, especially in scaphoid fractures. This trial is required to show the non-inferiority of magnesium-based screws compared to conventional titanium screws in hand and trauma surgery.

\section{Study objectives and hypotheses}

The general aim of this trial is to obtain precise, wellfounded and systematic application data of biodegradable magnesium-based compression screws compared to conventional titanium compression screws for the treatment of scaphoid fractures.

Specifically, we hypothesized that biodegradable magnesium-based compression screws are non-inferior compared with titanium screws in the treatment of isolated scaphoid fractures in terms of both efficacy and safety. In addition, we hypothesize that magnesium-based compression screws are superior to titanium screws in image analysis because magnesium-based compression screws are biodegradable.

The primary objective of this trial thus is to determine non-inferiority for efficacy and safety of magnesiumbased compression screws when compared to titanium screws in the surgical treatment of patients with scaphoid fracture. If non-inferiority can be shown for both efficacy and safety, the final primary aim is to demonstrate superiority of magnesium-based compression screws over titanium screws with the surrogate variable artifact reduction as indicator for degradation of magnesiumbased compression screws in-vivo.

Secondary objective of the trial is to compare quality of life in patients treated with magnesium-based compression screws with classical Herbert titanium screws.

\section{Methods}

\section{Study design}

SCAMAG is a randomized, controlled, parallel-group, open-label with blinded observer, multicenter trial with two groups for comparing biodegradable magnesiumbased screws with standard titanium Herbert screws. The study protocol follows the SPIRIT statement [29]. 
Reporting will follow the CONSORT statement [30] and its extension to abstracts [31].

Following the SPIRIT statement, we have created Additional file 1 to show the proposed participant flow through the study. The SPIRIT checklist is shown in Additional file 2.

The study was approved by the ethics committee of the Hanover Medical School (MHH) on September 27, 2017 (registration number: 7614), and it was registered with the German Register for Clinical Trials (DRKS, drks.de) on Dec 04, 2017 (registration number: DRKS00013368).

\section{Setting, recruitment, inclusion and exclusion criteria}

After diagnosis of a scaphoid fracture and when a decision for the indication of an osteosynthesis with compression screw is made, patients will be recruited by the surgeon in 13 high-volume centers in Germany. Each center has experience in conducting clinical trials, treating patients with scaphoid fractures and the use of both MAGNEZIX $^{\circ}$ CS compression screws and titanium Herbert screws. All surgeons are trained in fixation of scaphoid fractures with titanium Herbert screws and MAGNEZIX ${ }^{\bullet}$ CS compression screws prior to the trial.

Study centers will receive a case payment of $500 €$ in two parts. The first payment in the amount of $50 \%$ will be paid after surgery and the second part after 12 months, if complete follow-up data is available.

Inclusion and exclusion criteria are displayed in Table 1.

Table 1 Inclusion and exclusion criteria

Inclusion criteria:

- Indication for screw fixation of scaphoid fracture which is not older

than 12 weeks; classification by Herbert, modified by Krimmer of types

$\mathrm{A} 2, \mathrm{~B} 1, \mathrm{~B} 2, \mathrm{~B} 3$ [4],

- normal wrist function prior to fracture,

$\cdot$ age $\geq 18$ years,

- written informed consent for trial participation and surgery.

Exclusion criteria:

- Previous surgery on the wrist, associated injuries, state after or suspicion of complex regional pain syndrome (CRPS),

- simultaneous fractures of the forearm of both sides and those who will influence the postoperative care,

- known ligamentary concomitant injuries of the wrist on both sides and those who will influence the post-operative care,

- radiological findings of medium to high grade osteoporosis,

- intended or conducted spongiosa transplantation or bone graft

transplantation during surgery,

- pregnancy, suspected pregnancy or breastfeeding period,

- allergies to components of osteosynthesis material,

- participation in other clinical trials up to 30 days before inclusion in this trial,

- central neurological deficits which do not permit a compliance to the trial, especially during follow-up,

- for patients recruited to the centers near Hanover and undergoing MRI: claustrophobia and metallic implants which are contraindicative for an MRI.

\section{Intervention: magnesium-based screws}

Usual preoperative preparation will be performed. The biodegradable compression screw MAGNEZIX ${ }^{\circ}$ CS will be used in the following dimensions: MAGNEZIX ${ }^{\circ}$ CS $\varnothing 2.0 \mathrm{~mm}$, length $8 \mathrm{~mm}$ to $24 \mathrm{~mm}$ in $2 \mathrm{~mm}$ stages, cannulated MAGNEZIX ${ }^{\circ}$ CS $\varnothing 2.7 \mathrm{~mm}$, length $10 \mathrm{~mm}$ to 34 $\mathrm{mm}$ in $2 \mathrm{~mm}$ stages and cannulated MAGNEZIX ${ }^{\circ}$ CS $\varnothing$ $3.2 \mathrm{~mm}$, length $10 \mathrm{~mm}$ to $40 \mathrm{~mm}$ in $2 \mathrm{~mm}$ stages (Fig. 1).

Surgical procedures will be done as established in the study center with minimal access as possible: percutaneous, minimal invasive or open approach depending on the fracture type. After surgery the success of reposition and osteosynthesis will be controlled via plain radiographs. The further treatment and aftercare will be performed strictly as recommended in the AWMF S3 guideline for scaphoid fractures according to the fracture type: no immobilization for stable fractures, 4 weeks of immobilization for waist fractures and 6 weeks of immobilization for proximal pole fractures [6].

According to the fracture type splinting and training will be performed with $\mathrm{x}$-ray control. In case of unclear fracture healing as assessed by $\mathrm{x}$-rays, a CT-scan will be done to determine fracture healing but, according to the AWMF S3 guideline for scaphoid fractures [6] at the earliest 9 weeks after surgery.

\section{Control: titanium Herbert screws}

Usual preoperative preparation will be performed. Classical titanium Herbert screws will be used in the control group. Size of screws and manufacturer are chosen by the surgeon.

All other procedures are identical as in the magnesium group.

\section{Baseline and follow-up examinations}

An overview of scheduled study visits is shown in Table 2. A baseline examination will be prior to surgery after having obtained informed consent. Success of surgery will be checked, in general, at day 1 . Wound check is scheduled 2 weeks after surgery. Patients with scaphoid fractures grades B1 to B3 will be seen prior to exercise release. All patients will be seen prior to stress release. Time for exercise and stress release depend on the fracture type (Fig. 2). Before exercise release and before stress release after the period of exercising, clinical examinations will be performed.

Patients will be followed up at 3 months, 6 months and 12 months after randomization. Data will be collected by a physician and a nurse who will both be blind to the randomization status. The follow-up period will 12 months after randomization. 
Table 2 Schedule of assessments

\begin{tabular}{|c|c|c|c|c|c|c|c|c|c|}
\hline & Baseline & Surgery & Success of & Wound & Exercise & Stress & Follow-up & & \\
\hline & & & & & & & 3 months & 6 months & 12 months \\
\hline In- and exclusion criteria & $x$ & & & & & & & & \\
\hline Informed consent & $x$ & & & & & & & & \\
\hline Randomization & $x$ & & & & & & & & \\
\hline Medical history & $x$ & & & & & & & & \\
\hline Medical examination & $x$ & $x$ & $x$ & $x$ & $x$ & $x$ & $x$ & $x$ & $x$ \\
\hline Adverse events & & $x$ & $x$ & $x$ & $x$ & $x$ & $x$ & $x$ & $x$ \\
\hline EQ-5D-5 L & $x$ & & & & & & $x$ & $x$ & $x$ \\
\hline PRWE & & & & & & & $x$ & $x$ & $x$ \\
\hline DASH & & & & & & & $x$ & $x$ & $x$ \\
\hline KWS & & & & & & & $x$ & $x$ & $x$ \\
\hline X-ray / CT (according to guideline) & $x$ & & $x$ & & $x$ & $x$ & $(X)$ & & \\
\hline Range of motion & & & & & & $x$ & $x$ & $x$ & $x$ \\
\hline Grip strength & & & & & & $x$ & $x$ & $x$ & $x$ \\
\hline Pain NAS & & & $x$ & $x$ & $x$ & $x$ & $x$ & $x$ & $x$ \\
\hline
\end{tabular}

EQ-5D-5 L Quality of life; PRWE patient-rated wrist evaluation, DASH Disabilities of the Arm, Shoulder and Hand, KWS Krimmer Wrist Score, NAS Numeric analogue scale

\section{Magnetic resonance imaging (MRI) group of the trial}

Twenty randomized patients from the centers located in or near Hanover - 10 patients with titanium screws and 10 patients with MAGNEZIX ${ }^{\bullet} \mathrm{CS}$ - will additionally receive MRI imaging 6 weeks, 3, 6 and 12 months after randomization. MRI scans will be performed on a $3 \mathrm{~T}$ scanner located within the MHH.

The scanning protocol will include the following sequences: proton density weighted (PDw) turbo spin echo
(TSE) fat saturated (FS) sequences in coronal and axial planes (slice thickness $\leq 2 \mathrm{~mm}$ ), T2w TSE coronal (1 $\mathrm{mm}$, without FS) and sagittal $(\leq 2 \mathrm{~mm}, \mathrm{FS}), \mathrm{T} 1 \mathrm{w}$ TSE coronal ( $\leq 2 \mathrm{~mm}$, without FS) and a T1w TSE oblique sagittal sequence $(1 \mathrm{~mm}$, without $\mathrm{FS})$ adjusted to the scaphoid bone. There will be no gap between the slices and the field of view (FOV) will not exceed $10 \times 10 \mathrm{~cm}$. No intravenous contrast agent will be used. All images will be viewed in a preset window-level and width with

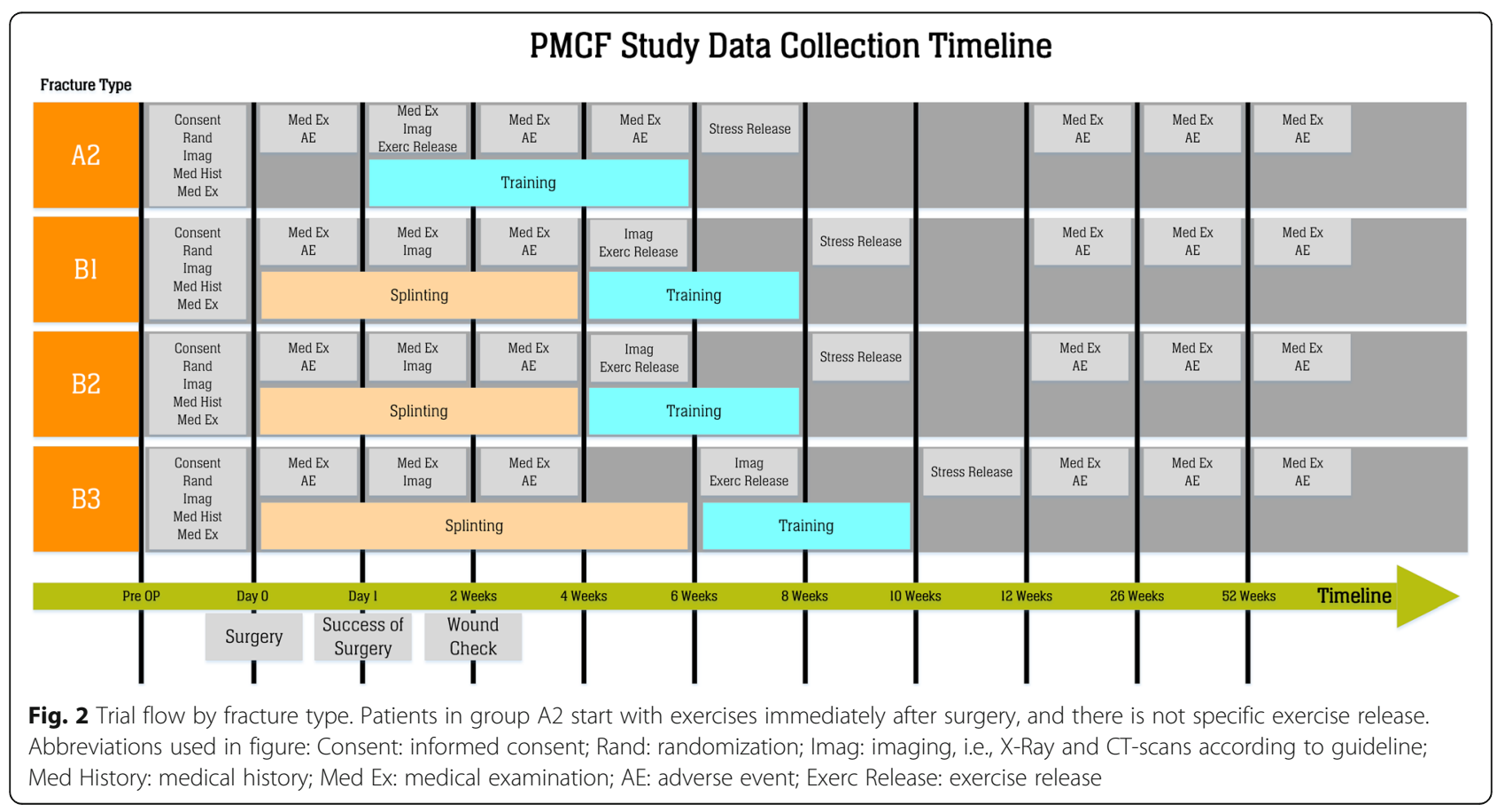


the following values for window center and width $(\mathrm{C} /$ W): PDw TSE/T1w 650/1300; PDw WARP 400/800.

\section{Primary endpoints}

Three primary endpoints will be used in this trial. The first primary outcome will be the German version (PRWE-G) of the patient-rated wrist evaluation (PRWE) total score measured 6 months after randomization [32]. The PRWE is a 15-item questionnaire which is completed by the patient. It is a brief, reliable and valid instrument for assessing wrist pain and disability [33, 34]. Scoring for all the questions is on a 10-point ordered scale ranging from 'no pain' or 'no difficulty' (0) to 'worst ever pain' or 'unable to do' (10). Two non-overlapping domains pain (5 items) and function (10 items) are generated. Pain and function scores are converted to scales of $0-50$ and are summed for a total score, which means that both domains are weighted equally. In case of a missing item, it has been recommended to replace the item with the mean score of the domain.

The PRWE has excellent test-retest reliability, validity and responsiveness [35]. Construct validity was significantly higher in PRWE compared to the short form 36 (SF-36) in patients with distal radius fracture, and it was similar to the DASH [36]. PRWE also outperformed the SF-36 in terms of responsiveness [37]. Overall, Dacombe et al. [35] concluded that the PRWE has by far the best demonstrated reliability and validity in a wrist trauma population among of all the patient rated outcome measures for the assessment of hand and wrist trauma. The PRWE has also been recommended as PRO by others, see, e.g., [38] They also concluded that the PRWE is the most responsive measure for a distal radius fracture population. It is used as primary outcome in at least two ongoing trials (ISRCTN67901257; NCT02154620).

Safety is the second primary outcome. It will be a composite endpoint, consisting of evidence of bone union until 6 months, no adverse device effect (ADE) during surgery, no ADE during wound healing, no reoperation and no serious adverse device effect (SADE) within 1 year after randomization. The outcome "safety event" thus is dichotomous and scored as 1 for a patient if

- an ADE occurred during surgery and/or

- an ADE occurred during the wound healing period and/or

- an SADE occurred during the 1-year follow-up period and/or

- a reoperation was performed during the 1-year follow-up period and/or

- there was no or incomplete bone union until 6 months after randomization, and 0 , otherwise.
AEs during surgery include the following:

- Nerve events of the superficial division of the radial nerve or the median nerve,

- vessel events with large hematoma with diameter $>2 \mathrm{~cm}$,

- screw related complications (fracture or bending of the screw),

- technical problems (protrusion into the adjacent joint and/or bone without correction, wrong choice of screw length) and

- revision of operation technique.

Artifacts will be measured as described by Sonnow et al. [15] to evaluate changes of artifact appearance over time. In detail, all artifacts will be assessed in an axial plane or reconstruction of the screw. In MRI artifacts aligning the $y$-axis will be considered, defined as the vertical axis of the scanner. As artifact appearance is expected to be symmetrical, measurement will be performed by creating a straight line through the outer boundaries of the artifacts and the central screw axis. The degree of artifact is defined as the diameter of the signal loss induced by the screw in MRI. When artifacts with various lengths are produced, the longest will be measured. This process will be performed in a total of three different axial slices of the screw, and the average value will be obtained. For better orientation and comparability, the artificial cartilage lesion will serve as reference, thus the slices in a similar position will be chosen.

The change in artifacts can now be defined as follows: Difference between maximum length of an artifact between 1-year follow-up and baseline. The third primary endpoint thus is defined quantitatively as the extent of change in artifacts. The specific imaging modality for the third primary endpoint will be defined as early as possible during the trial and before inclusion of the last patient of the MRI part of the trial.

\section{Secondary endpoints}

Secondary endpoints include the domains pain and function of the PRWE, measured at 3, 6 and 12 months. We also use the disabilities of the arm, shoulder and hand (DASH) questionnaire [39] - total score, module scores sport/music, module score work - and the Krimmer wrist score (KWS) with its four domains pain, active flexion/extension arc, grip strength and ability to return to regular employment or activities [40], both measured at 3 months, 6 months and 12 months as secondary endpoints. Range of motion will be assessed with a commercially available goniometer (wrist flexion, wrist extension, wrist radial deviation, wrist ulnar deviation, forearm supination, forearm pronation) at months 3,6 and 12 . At the same follow-up time points, grip strength will be determined with a dynamometer. 
Evidence of bone union until 6 months will be determined using $\mathrm{x}$-ray images in 2 planes: anterior-posterior and lateral, in case of uncertainty additionally in a third plane, namely Stecher. Union is defined as complete disappearance of the fracture line on radiographs. If bone healing cannot be assumed in plain radiographs, CTScan will be performed according to the AWMF guideline for scaphoid fractures [6]. Images 6 months after surgery will be assessed independently by two experienced radiologists. Edema in MRI will be recorded at 3, 6 and 12 months. Time until return to work and recreational activities will be established through patient self-report.

Finally, quality of life will be measured with the EQ5D-5 L [41, 42] at baseline, 3, 6 and 12 months after surgery.

\section{Blinding}

Blinded assessment will be done for KWS, range of motion (ROM) and grip strength. Blinding will not be possible for images. The PRWE is patient reported and therefore not assessable in a blinded fashion. In addition, blinding is not possible for the assessment of the effects of the devices for the safety endpoints.

\section{Randomization}

Patients will be randomized individually to one of the two treatment groups in a 1:1 ratio in the order they qualify. After inclusion and exclusion criteria have been checked, the presence of the informed consent form has been ticked and the form has been electronically signed by the surgeon, the randomization result will be displayed in the trial database after anesthesia. Permuted block randomization is used with variable block length stratified by center. Randomization lists for the PBR will be generated using the randomization software RITA [43]. Concealment of allocation will be guaranteed through central randomization within the electronic case report form (eCRF) according to Standard Operating Procedures (SOP).

An expert reviewer pointed out that the trial might have benefited from stratification by fracture type. The decision was to stratify by center because strata would have become very small in case of stratification by center and fracture type. Preference was given to center because of its known effect and its mentioning in the International Conference on Harmonisation (ICH) E9 guideline [44].

\section{Statistical analysis}

All statistical analyses will be described in detail in a statistical analysis plan (SAP) which will be finalized before the randomization of the last patient. Analysis populations for the primary endpoints will be the full analysis set (FAS) based on the intention to treat (ITT) principle and the per protocol population (PP). Neither interim analyses nor adaptations are planned for this trial. All statistical analyses will be done using the $R$ software.

The familywise error rate is set to $5 \%$. The three primary hypotheses will be investigated hierarchically.

The hypothesis for the first primary endpoint is that the mean PRWE in the magnesium-based group is non-inferior to the mean PRWE in the titanium group, measured 6 months after randomization. The non-inferiority margin is 10 points; low values of the PRWE denote 'no pain' or 'no difficulty'. The onesided $97.5 \%$ confidence interval from the t-test will be estimated for judging non-inferiority. Sensitivity analyses will be performed using linear mixed models with center as random effect.

The hypothesis for the second primary endpoint is that the rate of safety events in the magnesium-based group is non-inferior to that in the titanium group within 1 year after surgery. The non-inferiority margin is $15 \%$. The one-sided $97.5 \%$ Wilson score interval for the difference of two proportions will be estimated for judging non-inferiority [45]. Sensitivity analyses will be performed using logistic regression mixed models with center as random effect.

The type I error level is set to $2.5 \%$ one-sided for the first two primary endpoints. Non-inferiority will only be claimed if

1. the hypothesis for the first primary endpoint shows non-inferiority in the FAS based on the ITT population,

2. the hypothesis for the second primary endpoint shows non-inferiority in the FAS based on the ITT population,

3. the hypothesis for the first primary endpoint shows non-inferiority in the PP population and

4. the hypothesis for the second primary endpoint shows non-inferiority in the PP population.

No adjustments will be made for multiple testing because both tests need to demonstrate non-inferiority.

If non-inferiority has been established, superiority will be tested in the FAS based on the ITT population for the third primary endpoint. The hypothesis for the third primary endpoint is that the mean change in artifact size in MRI between 1-year follow-up and baseline in the magnesium-based group is different from the mean change in artifact size in MRI between 1-year follow-up and baseline in the titanium group. Superiority will be tested at the two-sided 5\% test level. The Welch-type ttest will be used for judging superiority at the two-sided $5 \%$ test-level. 
All secondary endpoints will be tested by appropriate tests and models exploratorily using the two-sided $5 \%$ significance level without adjustment for multiple testing.

Missing values for the primary endpoints will be imputed using MICE [46]. Missing data of scores from questionnaires will be handled according to the respective manual. As sensitivity analysis, a complete case analysis will be performed for the primary endpoints.

\section{Sample size calculations}

Aim of the trial is to demonstrate non-inferiority of magnesium-based compression screws when compared with titanium Herbert screws for the fixation of scaphoid fractures. Standard deviations (SD) for the PRWE in the literature were all approximately 20 points [47]. Lange and Freitag [48] reported in their systematic review that many studies used a non-inferiority margin of $0.5 \mathrm{SD}$. This limit is used a non-inferiority margin for the planning of the scaphoid trial for the primary endpoint PRWE. This leads to the following assumptions for the sample size calculations for the first primary endpoint: Allocation ratio 1:1, type I error level 0.025 one-sided, power 0.9 , expected difference between titanium and magnesium-based screws: $\Delta_{1}=0$, common standard deviation: $\sigma=20$, non-inferiority margin: $\Delta_{0}=10$, drop-out $10 \%$. To demonstrate non-inferiority under these assumptions, 94 patients per group, i.e., 188 patients in total are required for this trial.

If non-inferiority has been established for the first primary endpoint, the power to establish non-inferiority for the second primary endpoint, the safety endpoint is 90.64\% under the following assumptions: type I error level 0.025 one-sided, event rate for magnesium-based and titanium screws: 0.9 , non-inferiority margin: $\Delta_{0}=$ 0.15 , drop-out $10 \%$.

If non-inferiority has been established for the first primary endpoint and the second primary endpoint, the power to establish superiority for the third primary endpoint, the change in artifacts endpoint is virtually $100 \%$ under the following assumptions: type I error level 0.025 one-sided, expected difference between titanium and magnesium-based screws: $\Delta_{1}=3.0$, common standard deviation: $\sigma=1.0$, sample size per group: $n_{A}=n_{B}=10$, dropout $10 \%$, effective sample size per group: $n_{A}=n_{B}=9$. The power is $56.41 \%$ if the mean difference is $\Delta_{1}=1.0$ instead of $\Delta_{1}=3.0$, and it is approximately $80 \%$ for $\Delta_{1}=1.32$.

The expected difference between the titanium and the magnesium-based group are substantiated as follows. Mean \pm standard deviation artifact size were $8.4 \pm 0.7$ $\mathrm{mm}$ in the magnesium group and $12.9 \pm 1.0 \mathrm{~mm}$ in the titanium group in the work of Sonnow et al. [15]. We assume a random reduction in artifact size in the titanium group of $1.0 \mathrm{~mm}$ and a halving of the artifact size in the magnesium group so that the difference is $3.4 \mathrm{~mm}$, which is rounded to $3 \mathrm{~mm}$. Due to the lack of additional data, we assume a common standard deviation of $1 \mathrm{~mm}$, which is the standard deviation in the titanium group.

\section{Data management and data monitoring}

Database and eCRF are developed, maintained and hosted by AMEDON GmbH. AMEDON GmbH will also perform data management. The trial database has been developed and validated before data entry based on standard operating procedures (SOPs). All changes made to the data are documented in an audit trail. The trial software has a user and role concept that can be adjusted on a trial-specific basis. The database is integrated into a general information technology infrastructure and safety concept with a firewall and backup system. The data are backed up daily. After completion and cleaning of data, the database is locked, and the data are exported for statistical analysis.

The data will be entered via the internet at the trial sites. Plausibility checks are run during data entry, thereby detecting many discrepancies immediately. Data management and monitoring will conduct further checks for data completeness and plausibility of data, and they will clarify any questions with the trial sites. These queries must be answered by the trial site without unreasonable delay. Further details are specified in the Data Validation Plan and in the Monitoring Manual.

Data management will be performed in compliance with AMEDON GmbH and in accordance to EN 14155 [49].

All relevant study data will be stored by the sponsor of the study. In the individual participating centers, the investigator files, study-related correspondence, patient identification list, consent forms and patient files will be retained for at least 10 years after the end of the study. Other in-house regulations or legislations demanding longer retention periods (e.g. radiation control regulation, radiation protection law) will be respected.

AMEDON $\mathrm{GmbH}$ is responsible for clinical onsite monitoring according to EN14155, written SOPs and the monitoring manual to ensure patient's rights, patient's security and reliability of trial results. For initiation, the trial site will be visited onsite by a clinical research associate. During the trial, sites will be visited at regular intervals depending on the rate of recruiting and data quality.

No audits are planned. However, to ensure correct execution of the study, audits may be conducted if necessary.

The trial is under medical direction. All staff members underlie medical confidentiality. Within a first training of staff and additional, consistently occurring staff trainings, the authorized clinic staff in the study sites will be 
instructed to handle all data as well as project-specific contents confidentially and to use all regular protection measures (recent version of antivirus-software, computer blocking by leaving the room, logout from eCRF after successful data transfer etc.).

\section{Governance}

The whole project is supervised by a steering committee, which has regular meetings over the phone. An independent data monitoring committee (DMC) was not established because of the expected short accrual time. As the current study is conducted according to $\$ 23 \mathrm{~b}$ of the German medical product act, no inspections of higher federal authorities are scheduled.

\section{Discussion}

Scaphoid fractures are often fixated using titanium Herbert screws, which generally remain in the body. However, allergic reactions against titanium implants have been reported in few cases only [50, 51]. More important is screw removal which requires a second surgery. Biodegradable implants have the advantage that a second surgery for implant removal is not required. Polymer screws are biodegradable but their stability is substantially lower than the stability of titanium screws $[8,52,53]$. Magnesium-based screws combine the advantages of both materials, high stability together with biodegradability. MAGNEZIX ${ }^{\bullet}$ CS compression screws are the world's first magnesiumbased implants designed for use in biodegradable osteosyntheses applications in humans with market approval for the European Union and other countries [9]. An economic analysis for magnesium-based implants has been provided recently [21].

The non-inferiority of magnesium-based when compared with titanium compression screws has been demonstrated for hallux valgus surgery in a randomized controlled trial [20]. However, for scaphoid fractures only case reports have been published in which the use of magnesium-based implants is described [26, 27]. The randomized controlled SCAMAG trial will therefore generate high-level evidence in the use of magnesium-based compression screws for the treatment of scaphoid fractures.

\section{Trial status}

The trial opened for accrual on Jan 2, 2018. First patient in was on Jan 4, 2018. Accrual is planned to be completed by the end of 2020 .

\section{Additional files}

Additional file 1: Figure S1. Study flow according to CONSORT statement. (PDF $1348 \mathrm{~kb}$ )
Additional file 2: SPIRIT 2013 and SPIRIT 2018 PRO Checklist applied to SCAMAG study protocol. (DOC $179 \mathrm{~kb})$

\section{Abbreviations}

ADE: Adverse Device Effect; AE: Adverse Event; CONSORT: Consolidated Standards of Reporting Trials; CT: Computed Tomography; DASH: Disabilities of the Arm, Shoulder and Hand; DASH: Disability of the Arm, Shoulder and Hand; eCRF: Electronic Case Report Form; EN: European Norm; EQ-5D-5 L: Quality of life questionnaire; FAS: Full Analysis Set; FOV: Field Of View; FS: Fat saturated; GCP: Good Clinical Practice; ICH: International Conference on Harmonisation; ITT: Intention To Treat; KWS: Krimmer Wrist Score; MRI: Magnetic Resonance Imaging; PDw: Proton Density weighted; PP: Per Protocol; PRWE: Patient-Rated Wrist Evaluation; SADE: Serious Adverse Device Effect; SAE: Serious Adverse Event; SAP: Statistical Analysis Plan; SD: Standard Deviation; SOP: Standard Operating Procedure; SPIRIT: Standard Protocol Items: Recommendations for Interventional Trials; TSE: Turbo spin echo; WARP: Summary of methods to minimize the impact of metal implants on image quality

\section{Acknowledgements}

Not applicable.

\section{Authors' contributions}

$\mathrm{MHK}, \mathrm{SK}, \mathrm{CP}, \mathrm{PV}$ and AZ conceived the study. They initiated the study design, and $\mathrm{KK}$ and $\mathrm{BB}$ helped with the implementation. SK and CVF designed the image analysis protocol of the trial. AZ provided statistical expertise in clinical trial design, is responsible for the PRO content and drafted the manuscript. All authors contributed to the refinement of the study protocol, reviewed and approved the final manuscript.

\section{Funding}

This trial is fully financed by Syntellix AG. The Syntellix AG was involved in the study design.

\section{Availability of data and materials}

Additional information on the study protocol will not be available because all relevant information is provided in this manuscript.

Ethics approval and consent to participate

Ethics approval was obtained from the ethics committee of the Medizinische Hochschule Hannover (MHH) on September 27, 2017, and its registration number is 7614 .

Written informed consent for trial participation will be obtained from all patients prior to randomization.

Consent for publication

Not applicable.

\section{Competing interests}

CVF, SK and PMV are employees of MHH, which receives a grant from Syntellix AG for conducting the trial. CP is an employee of Syntellix AG, and MHK is board member of Syntellix AG. KK is an employee of AMEDON $\mathrm{GmbH}$, and BB is CEO of AMEDON GmbH. AMEDON GmbH provides trial services to Syntellix AG. AZ is a consultant to Syntellix AG.

\section{Author details}

'Department of Plastic, Aesthetic, Hand and Reconstructive Surgery, Hanover Medical School (MHH), Carl-Neuberg-Straße 1, 30625 Hannover, Germany. ${ }^{2}$ Amedon GmbH, Willy-Brandt-Allee 31c, 23554 Lübeck, Germany. ${ }^{3}$ Syntellix AG, Aegidientorplatz 2a, 30159 Hannover, Germany. ${ }^{4}$ Institute for Diagnostic and Interventional Radiology, Hanover Medical School, Carl-Neuberg-Str. 1, 30625 Hannover, Germany. ${ }^{5}$ Department for General, Trauma and Reconstructive Surgery, Ludwig-Maximilians University of Munich, Marchioninistraße 15, 81377 Munich, Germany. ${ }^{6}$ StatSol, Moenring 2, 23560 Lübeck, Germany. ${ }^{7}$ School of Mathematics, Statistics and Computer Science, University of KwaZulu-Natal, Pietermaritzburg, South Africa. 
Received: 23 January 2019 Accepted: 26 June 2019 Published online: 07 August 2019

\section{References}

1. Hove LM. Epidemiology of scaphoid fractures in Bergen, Norway. Scand J Plast Reconstr Surg Hand Surg. 1999;33:423-6.

2. Holloway KL, Moloney DJ, Brennan-Olsen SL, Kotowicz MA, Bucki-Smith G, Morse AG, Timney EN, Dobbins AG, Hyde NK, Pasco JA. Carpal and scaphoid fracture incidence in South-Eastern Australia: an epidemiologic study. Arch Osteoporos. 2015;10:10.

3. Amirfeyz R, Bebbington A, Downing ND, Oni JA, Davis TR. Displaced scaphoid waist fractures: the use of a week 4 CT scan to predict the likelihood of union with nonoperative treatment. J Hand Surg Eur Vol. 2011;36:498-502.

4. Krimmer $\mathrm{H}$, Schmitt R, Herbert T. [scaphoid fractures-diagnosis, classification and therapy] Kahnbeinfrakturen - Diagnostik, Klassifikation und Therapie. Unfallchirurg. 2000;103:812-9.

5. Suh N, Grewal R. Controversies and best practices for acute scaphoid fracture management. J Hand Surg Eur Vol. 2018;43:4-12.

6. Deutsche Gesellschaft für Unfallchirurgie. [AWMF guideline 012-016: scaphoid fracture] AWMF-Leitlinie 012 - 016: Skaphoidfraktur. Düsseldorf: AWMF; 2015.

7. Wang $Y$, Song $M, X u Y$, He $X$, Zhu Y. Absorbable scaphoid screw development: a comparative study on biomechanics. Ther Clin Risk Manag. 2016;12:643-50.

8. Seitz J-M, Durisin M, Goldman J, Drelich JW. Recent advances in biodegradable metals for medical sutures: a critical review. Adv Healthcare Mater. 2015;4: 1915-36.

9. Seitz J-M, Lucas A, Kirschner M. Magnesium-based compression screws: a novelty in the clinical use of implants. JOM. 2016;68:1177-82.

10. Wild M, Windolf J, Flohé S. [Fractures of the patella] Patellafrakturen. Unfallchirurg. 2010;113:401-11.

11. Mayer G, Seidlein H. Chondral and osteochondral fractures of the knee joint--treatment and results. Arch Orthop Trauma Surg. 1988;107:154-7.

12. Sie TH, Abdel-Kader KF, Allcock S. A useful technique for removal of Herbert screws from the scaphoid. J Hand Surg Br. 1998;23:332-3.

13. Radford PJ, Matthewson MH, Meggitt BF. The Herbert screw for delayed and non-union of scaphoid fractures: a review of fifty cases. J Hand Surg Br. 1990;15:455-9.

14. Gregory JJ, Mohil RS, Ng AB, Warner JG, Hodgson SP. Comparison of Herbert and Acutrak screws in the treatment of scaphoid non-union and delayed union. Acta Orthop Belg. 2008;74:761-5.

15. Sonnow L, Konneker S, Vogt PM, Wacker F, von Falck C. Biodegradable magnesium Herbert screw - image quality and artifacts with radiography, CT and MRI. BMC Med Imaging. 2017;17:16.

16. Andrade VM, Aschner M, Marreilha Dos Santos AP. Neurotoxicity of metal mixtures. Adv Neurobiol. 2017;18:227-65.

17. Waizy H, Diekmann J, Weizbauer A, Reifenrath J, Bartsch I, Neubert V, Schavan $\mathrm{R}$, Windhagen $\mathrm{H}$. In vivo study of a biodegradable orthopedic screw (MgYREZr-alloy) in a rabbit model for up to 12 months. J Biomat Applicat. 2014;28:667-75.

18. Waizy H, Seitz JM, Reifenrath J, Weizbauer A, Bach FW, Meyer-Lindenberg A, Denkena B, Windhagen H. Biodegradable magnesium implants for orthopedic applications. J Mater Sci. 2013;48:39-50.

19. Plaaß C, von Falck C, Ettinger S, Sonnow L, Calderone F, Weizbauer A, Reifenrath J, Claassen L, Waizy H, Daniilidis K, et al. Bioabsorbable magnesium versus standard titanium compression screws for fixation of distal metatarsal osteotomies - 3 year results of a randomized clinical trial. J Orthop Sci. 2018;23:321-7.

20. Windhagen H, Radtke K, Weizbauer A, Diekmann J, Noll Y, Kreimeyer U, Schavan R, Stukenborg-Colsman C, Waizy H. Biodegradable magnesiumbased screw clinically equivalent to titanium screw in hallux valgus surgery: short term results of the first prospective, randomized, controlled clinical pilot study. BioMed Engineer OnLine. 2013;12:62.

21. Klauser $\mathrm{H}$. Internal fixation of three-dimensional distal metatarsal I osteotomies in the treatment of hallux valgus deformities using biodegradable magnesium screws in comparison to titanium screws. Foot Ankle Surg. 2018; in press.

22. Plaaß C, Ettinger S, Sonnow L, Könneker S, Noll Y, Weizbauer A, Reifenrath J, Claassen L, Daniilidis K, Stukenborg-Colsman C, Windhagen H. Early results using a biodegradable magnesium screw for modified chevron osteotomies. J Orthop Res. 2016;34:2207-14.

23. Acar B, Unal M, Turan A, Kose O. Isolated lateral malleolar fracture treated with a bioabsorbable magnesium compression screw. Cureus. 2018;10:e2539.
24. Kose O, Turan A, Unal M, Acar B, Guler F. Fixation of medial malleolar fractures with magnesium bioabsorbable headless compression screws: short-term clinical and radiological outcomes in eleven patients. Arch Orthop Trauma Surg. 2018;138:1069-75.

25. Biber R, Pauser J, Brem M, Bail HJ. Bioabsorbable metal screws in traumatology: a promising innovation. Trauma Case Reports. 2017;8:11-5.

26. Kirschner MH, Seitz JM. [The use of bioabsorbable magnesium-based implants in orthopedic surgery] Der Einsatz bioabsorbierbarer, magnesiumbasierter Implantate in der Knochen-Chirurgie. Wehrmed Wehrpharm. 2016; 2016(2):76.

27. Grieve P, O'Carroll S, Albastaki O. Six cas de série de patients de Magnezix ${ }^{\odot}$. Une vis métallique absorbable pour la fixation de la fracture du carpe et des fusions entre les carpes. Hand Surg Rehab. 2017;36:488-9.

28. Biber R, Pauser J, Gesslein M, Bail HJ. Magnesium-based absorbable metal screws for intra-articular fracture fixation. Case Rep Orthop. 2016;2016: 9673174.

29. Chan AW, Tetzlaff JM, Altman DG, Laupacis A, Gøtzsche PC, Krleža-Jerić K, Hróbjartsson AA, Mann H, Dickersin K, Berlin JA, et al. SPIRIT 2013 statement: defining standard protocol items for clinical trials. Ann Intern Med. 2013;158: 200-7.

30. Schulz KF, Altman DG, Moher D, for the CONSORT Group. CONSORT 2010 statement: updated guidelines for reporting parallel group randomised trials. PLoS Med. 2010;7:e1000251.

31. Hopewell S, Clarke M, Moher D, Wager E, Middleton P, Altman DG, Schulz KF. CONSORT for reporting randomised trials in journal and conference abstracts. Lancet. 2008;371:281-3.

32. John M, Angst F, Awiszus F, Pap G, Macdermid JC, Simmen BR. The patient-rated wrist evaluation (PRWE): cross-cultural adaptation into German and evaluation of its psychometric properties. Clin Exp Rheumatol. 2008;26:1047-58.

33. MacDermid JC, Turgeon T, Richards RS, Beadle M, Roth JH. Patient rating of wrist pain and disability: a reliable and valid measurement tool. J Orthop Trauma. 1998;12:577-86.

34. MacDermid JC. Development of a scale for patient rating of wrist pain and disability. J Hand Ther. 1996;9:178-83.

35. Dacombe PJ, Amirfeyz R, Davis T. Patient-reported outcome measures for hand and wrist trauma: is there sufficient evidence of reliability, validity, and responsiveness? Hand. 2016;11:11-21.

36. Lövgren A, Hellström K. Reliability and validity of measurement and associations between disability and behavioural factors in patients with Colles' fracture. Physiother Theory Pract. 2012;28:188-97.

37. MacDermid JC, Richards RS, Donner A, Bellamy N, Roth JH. Responsiveness of the short form-36, disability of the arm, shoulder, and hand questionnaire, patient-rated wrist evaluation, and physical impairment measurements in evaluating recovery after a distal radius fracture. J Hand Surg Am. 2000;25:330-40.

38. Calfee RP, Adams AA. Clinical research and patient-rated outcome measures in hand surgery. J Hand Surg Am. 2012;37:851-5.

39. Hudak PL, Amadio PC, Bombardier C. Development of an upper extremity outcome measure: the DASH (disabilities of the arm, shoulder and hand). Am J Ind Med. 1996;29:602-8.

40. Krimmer $\mathrm{H}$, Wiemer $\mathrm{P}$, Kalb K. [Comparative outcome assessment of the wrist joint-mediocarpal partial arthrodesis and total arthrodesis] Vergleichende Ergebnisbewertung am Handgelenk - mediokarpale Teilarthrodese und Totalarthrodese. Handchir Mikrochir Plast Chir. 2000; 32:369-74.

41. Brooks R. EuroQol: the current state of play. Health Policy. 1996;37:53-72.

42. Devlin NJ, Brooks R. EQ-5D and the EuroQol group: past, present and future. Appl Health Econ Health Policy. 2017;15:127-37.

43. Pahlke F, König IR, Ziegler A. [randomization in treatment arms (RITA): a randomization program for clinical trials] randomization in treatment arms (RITA): Ein Randomisierungs-Programm für klinische Studien. Inform Biom Epidemiol Med Biol. 2004;35:1-22.

44. CPMP: ICH topic E 9. Statistical principles for clinical trials. Note for guidance on statistical principles for clinical trials. CPMP/ICH/363/96. 1998. https:// www.ich.org/fileadmin/Public_Web_Site/ICH_Products/Guidelines/Efficacy/ E9/Step4/E9_Guideline.pdf.

45. Newcombe RG. Interval estimation for the difference between independent proportions: comparison of eleven methods. Stat Med. 1998;17:873-90.

46. Azur MJ, Stuart EA, Frangakis C, Leaf PJ. Multiple imputation by chained equations: what is it and how does it work? Int J Methods Psychiatr Res. 2011;20:40-9. 
47. Sorensen AA, Howard D, Tan WH, Ketchersid J, Calfee RP. Minimal clinically important differences of 3 patient-rated outcomes instruments. J Hand Surg. 2013;38:641-9.

48. Lange S, Freitag G. Choice of delta: requirements and reality--results of a systematic review. Biom J. 2005;47:12-27 discussion 99-107.

49. European Standard BS EN ISO 14155:2012-01: Clinical investigation of medical devices for human subjects. Good clinical practice. https://www. beuth.de/de/norm/din-en-iso-14155/134954877.

50. Wang L-F, Wu J, Zheng C, Li S-L, Huang R-R, Zhang J-K. Long-term fever after hallux valgus surgery secondary to titanium allergy: a case report and review of the literature. J Foot Ankle Surg. 2016;55:1282-6.

51. Thomas $P$, Thomas $M$, Summer B, Dietrich K, Zauzig M, Steinhauser E, Krenn $V$, Arnholdt H, Flaig MJ. Impaired wound-healing, local eczema, and chronic inflammation following titanium osteosynthesis in a nickel and cobaltallergic patient: a case report and review of the literature. J Bone Joint Surg Am. 2011;93:e61.

52. Witte F, Hort N, Vogt C, Cohen S, Kainer KU, Willumeit R, Feyerabend F Degradable biomaterials based on magnesium corrosion. Curr OpinSolid State Mat Sci. 2008;12:63-72.

53. Ezechieli M, Ettinger M, Konig C, Weizbauer A, Helmecke P, Schavan R, Lucas A, Windhagen $\mathrm{H}$, Becher $\mathrm{C}$. Biomechanical characteristics of bioabsorbable magnesium-based (MgYREZr-alloy) interference screws with different threads. Knee Surg Sports Traumatol Arthrosc. 2016;24: 3976-81.

\section{Publisher's Note}

Springer Nature remains neutral with regard to jurisdictional claims in published maps and institutional affiliations.

Ready to submit your research? Choose BMC and benefit from:

- fast, convenient online submission

- thorough peer review by experienced researchers in your field

- rapid publication on acceptance

- support for research data, including large and complex data types

- gold Open Access which fosters wider collaboration and increased citations

- maximum visibility for your research: over $100 \mathrm{M}$ website views per year

At $\mathrm{BMC}$, research is always in progress.

Learn more biomedcentral.com/submissions 